\title{
Modeling and System Identification of the muFly Micro Helicopter
}

\section{Journal Article}

Author(s):

Schafroth, Dario; Bermes, Christian; Bouabdallah, Samir; Siegwart, Roland

Publication date:

2010-01

Permanent link:

https://doi.org/10.3929/ethz-b-000017462

Rights / license:

In Copyright - Non-Commercial Use Permitted

Originally published in:

Journal of Intelligent \& Robotic Systems 57(1-4), https://doi.org/10.1007/s10846-009-9379-x 


\title{
Modeling and System Identification of the muFly Micro Helicopter
}

\author{
Dario Schafroth • Christian Bermes • \\ Samir Bouabdallah · Roland Siegwart
}

Received: 1 February 2009 / Accepted: 1 August 2009 / Published online: 27 October 2009

(c) Springer Science + Business Media B.V. 2009

\begin{abstract}
An accurate mathematical model is indispensable for simulation and control of a micro helicopter. The nonlinear model in this work is based on the rigid body motion where all external forces and moments as well as the dynamics of the different hardware elements are discussed and derived in detail. The important model parameters are estimated, measured or identified in an identification process. While most parameters are identified from test bench measurements, the remaining ones are identified on subsystems using the linear prediction error method on real flight data. The good results allow to use the systems for the attitude and altitude controller design.
\end{abstract}

Keywords Coaxial micro helicopter $\cdot$ Nonlinear model $\cdot$ Parameter identification

\section{Introduction}

The European Framework project muFly has been initiated to develop a fully autonomous micro helicopter designated for tasks such as surveillance and security, search and rescue, as well as inspection and exploration. Target capabilities of the system are autonomous take off/hover/landing, obstacle avoidance and way point following. For such capabilities a fast and precise feedback control is needed on the low level, which makes an appropriate simulation model of the helicopter indispensable for the controller design. While there exist different other works on modeling conventional $[1,2]$ and coaxial helicopters $[3,4]$ it is necessary to adapt the model to the specialties of the used platform. One of those specialties is the stabilizer bar mounted on the helicopter, which passively stabilizes the system and

D. Schafroth $(\varangle) \cdot$ C. Bermes $\cdot$ S. Bouabdallah $\cdot$ R. Siegwart Autonomous System Lab, Swiss Federal Institute of Technology Zurich (ETHZ), Zurich, Switzerland e-mail: sdario@ethz.ch 
strongly influences the dynamics. Further devices, such as the electro motor and drive train are not considered in the found works, but are important for the designated control tasks. Therefore, a custom-made dynamic model is developed for muFly. The goal is to have a structured model reflecting accurately the physics of the different subsystems for simulations, but on the other side be as simple as possible since it will be used for the controller design later on.

The nonlinear model is based on the rigid body motions, where all the existing external forces and moments are discussed and derived. This is followed by defining the dynamics of the single mechanical devices such as swash plate, electro motors or stabilizer bar to complete the model. The developed model is then verified on real flight data and the system parameters are identified. This process is very important since a model with unknown or wrong parameters is worthless. For simplification the system identification is done on the linearized model using the prediction error method (PEM) [5]. Linearization allows to decouple the model in different subsystems and therefore strongly reduces the complexity. Using the linearized model is adequate since muFly will only operate close to the hover point. High velocities are not foreseen for the missions.

The paper is organized as follows. In Section 2 the muFly micro helicopter and its hardware setup is shown, followed by the nonlinear model in Section 3. The identification process with the PEM is presented in Section 4 and the identification and verification results are shown in Section 5.

\section{The muFly Helicopter}

muFly is a $17 \mathrm{~cm}$ in span, $15 \mathrm{~cm}$ in height coaxial helicopter with a mass of $95 \mathrm{~g}$ (Fig. 1). The two main rotors are driven by two lightweight brushless DC (BLDC)

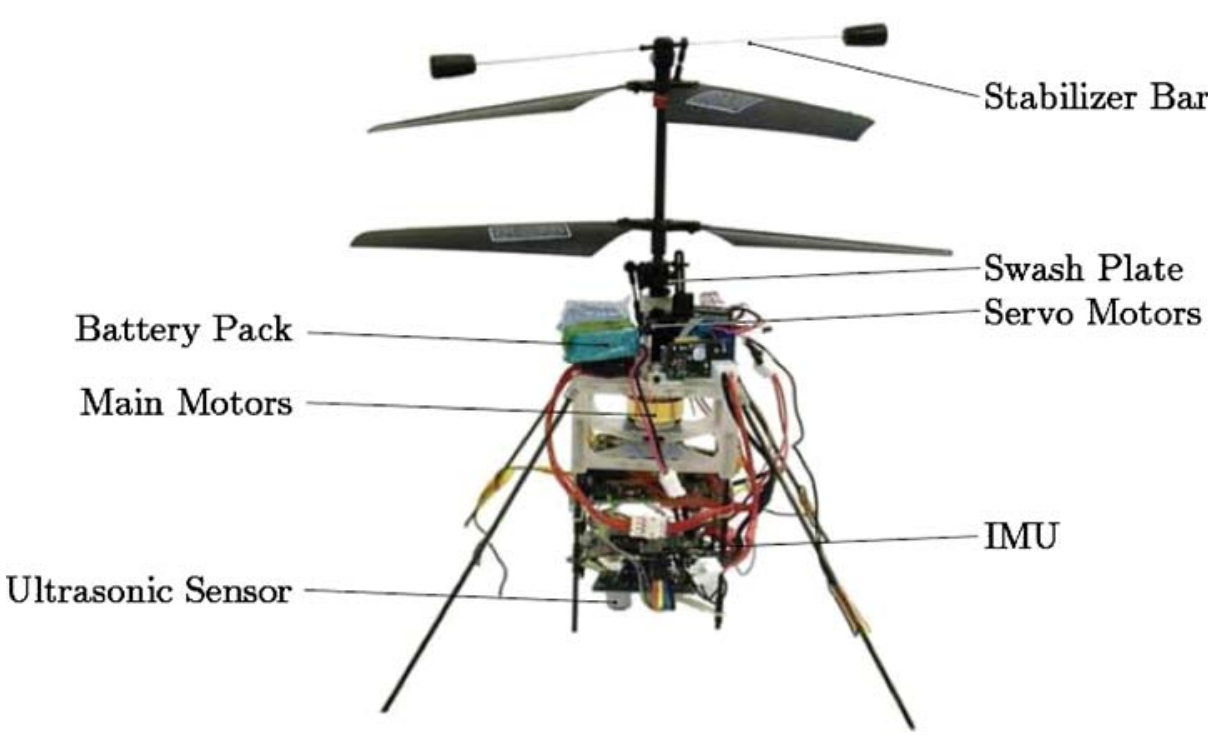

Fig. 1 The second prototype of the muFly helicopter 
motors and are counter rotating to compensate the resulting torque due to aerodynamical drag. This allows to control the yaw by differential speed variation of the two rotors, where as the altitude can be controlled varying the rotor speed simultaneously. The motor speed is reduced by a gear to achieve a higher torque on the rotor side.

A benefit of the coaxial setup is that one rotor can be used to help stabilizing the helicopter using a stabilizer bar. Such devices are often found on RC-Models and is mounted on the upper rotor. The helicopter is steered by a conventional swash plate actuated by two servos and powered by a lithium polymer battery. All the signals to the servos and motor controllers are pulse position modulated (PPM) signals, scaled to $u_{\text {serv }}=\{-1,1\}$ and $u_{\text {mot }}=\{0,1\}$.

Sensors mounted on the platform are an inertial measurement unit (IMU) and an ultrasonic distance sensor for the measurement of the distance to the ground. With this setup, the attitude angles and the height over ground can be measured. Further, an omnidirectional camera in combination with lasers is in development, that will be used for the measurement of the horizontal position. The sensor data is processed by a dsPIC microprocessor and send to the ground station by a serial connection using a bluetooth module. To minimize time delays, the actuator inputs are send in the same package as the sensor data.

\section{Nonlinear Modeling}

The goal of the model presented in this paper is to be as simple as possible, since it will be used for the controller design. On the other hand, the physics and dynamics of the different devices mounted on the muFly helicopter have to be reflected accurately. The complete nonlinear model is described in the following sections.

\subsection{Coordinate Frames, Kinematics and Dynamics}

As common two coordinate frames, the inertial frame $J$ and the body-fixed frame $B$ are defined for the model. Where as the body-fixed frame is placed in the Center of Gravity ( $\mathrm{CoG})$ and moves with the helicopter, the inertial frame is fixed to the original location of the body-fixed frame. The frames are defined as it is common in aviation and are shown in the schematic view of the helicopter in Fig. 2.

Corresponding to these definitions the transformation from the inertial frame $J$ to the body-fixed frame $B$

$$
\left[\begin{array}{c}
x \\
y \\
z
\end{array}\right]=\underline{\underline{\mathbf{A}}}_{B J} \cdot\left[\begin{array}{c}
N \\
E \\
D
\end{array}\right]
$$

is given by the transformation matrix

$$
\underline{\underline{\mathbf{A}}}_{B J}=\left[\begin{array}{ccc}
\mathrm{c} \theta \mathrm{c} \psi & \mathrm{c} \theta \mathrm{s} \psi & -\mathrm{s} \theta \\
-\mathrm{c} \phi \mathrm{s} \psi+\mathrm{s} \phi \mathrm{s} \theta \mathrm{c} \psi & \mathrm{c} \phi \mathrm{c} \psi+\mathrm{s} \phi \mathrm{s} \theta \mathrm{s} \psi & \mathrm{s} \phi \mathrm{c} \theta \\
\mathrm{s} \theta \mathrm{s} \psi+\mathrm{c} \phi \mathrm{s} \theta \mathrm{c} \psi & -\mathrm{s} \phi \mathrm{c} \psi+\mathrm{c} \phi \mathrm{s} \theta \mathrm{s} \psi & \mathrm{c} \phi \mathrm{c} \theta
\end{array}\right]
$$

obtained by the application of the Euler angles $(c \alpha=\cos (\alpha)$ and $\operatorname{s} \alpha=\sin (\alpha))$. 
Fig. 2 Coordinate frame convention and the forces and moments acting in hover (back view)
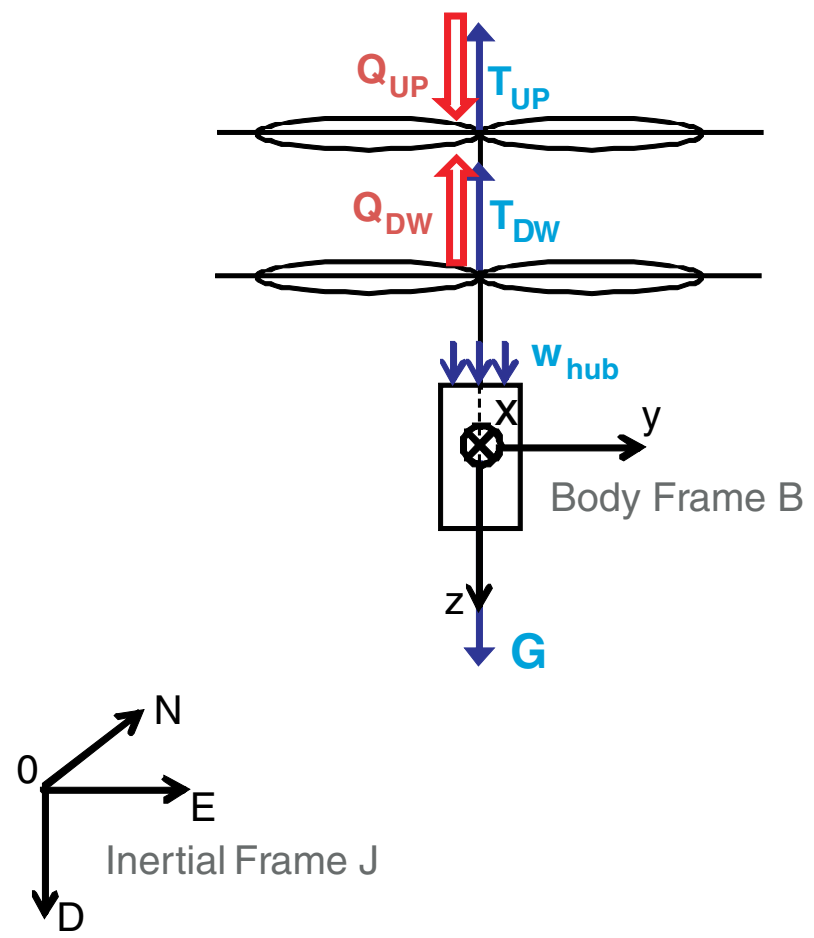

It is well known that this transformation matrix is not valid for angular quantities. Therefore the angular velocities $p, q, r$ have to be transformed using the transformation matrix

$$
\underline{\underline{\mathbf{R}}}_{J B}=\left[\begin{array}{ccc}
1 & \frac{\mathrm{s} \phi \mathrm{s} \theta}{\mathrm{s} \theta} & \frac{\mathrm{s} \phi \mathrm{s} \theta}{\mathrm{s} \theta} \\
0 & \mathrm{~s} \phi & -\mathrm{s} \phi \\
0 & \frac{\mathrm{s} \phi}{\mathrm{s} \theta} & \frac{\mathrm{s} \phi}{\mathrm{s} \theta}
\end{array}\right]
$$

that can be used to obtain a differential equation for the time derivatives of the roll, pitch and yaw angles $\phi, \theta$ and $\psi$ :

$$
\left[\begin{array}{c}
\dot{\phi} \\
\dot{\theta} \\
\dot{\psi}
\end{array}\right]=\underline{\underline{\mathbf{R}}}_{J B} \cdot\left[\begin{array}{c}
p \\
q \\
r
\end{array}\right] .
$$

The next step is to set up the rigid body dynamics equations in the CoG in the body fixed coordinate frame. Using Newtonian Mechanics, the differential equations for the rigid body motion in the body-fixed frame become

$$
\left[\begin{array}{c}
\dot{u} \\
\dot{v} \\
\dot{w}
\end{array}\right]=\frac{1}{m} \mathbf{F}-\left[\begin{array}{c}
p \\
q \\
r
\end{array}\right] \times\left[\begin{array}{c}
u \\
v \\
w
\end{array}\right]
$$


and

$$
\left[\begin{array}{c}
\dot{p} \\
\dot{q} \\
\dot{r}
\end{array}\right]=\underline{\mathbf{I}}^{-1}\left(\mathbf{M}-\left[\begin{array}{c}
p \\
q \\
r
\end{array}\right] \times \underline{\mathbf{I}}\left[\begin{array}{l}
p \\
q \\
r
\end{array}\right]\right)
$$

with the body velocities $u, v, w$, the system mass $m$, the body inertia tensor $\mathbf{I}$ and the total external force and moment vectors $\mathbf{F}$ and $\mathbf{M}$. For simplification, the inertia tensor I has only diagonal elements. Such a simplification is feasible as a result of the symmetric design of muFly.

So far the equations of motion are independent of the flying platform and can be found in literature [2]. Now the platform dependent total external force $\mathbf{F}$ and moment $\mathbf{M}$ have to be defined.

\subsection{Forces and Moments}

In a stable hover position, as shown in Fig. 2, the thrust forces from the two rotors $T_{\text {up }}$ and $T_{\mathrm{dw}}$ equal the gravitational force $G$ caused by the mass of the helicopter and the integrated aerodynamical drag force on the fuselage $W_{\text {hub }}$ due to the down wash of the rotors. The moments acting on the helicopter are the two drag torques $Q_{\text {up }}$ and $Q_{\mathrm{dw}}$ from the counter rotating rotors (incl. stabilizer bar), which, if unbalanced, lead to a yaw motion of the helicopter.

In free flight, additional forces and moments result from the aerodynamical drag due to the motion through the air, but since the helicopter is small and reaches only low velocities, those can be neglected. More important are the moments resulting from the tilting of the tip path plane (TPP) - the plane the rotor blade tips are running in [6] - on the lower rotor caused by a cyclic pitch input from the swash plate [7] and on the upper rotor due to the stabilization mechanism. These moments are used for the steering and stabilization of the helicopter.

Summarized, the force vector $\mathbf{F}$ and moment vector $\mathbf{M}$ are

$$
\begin{aligned}
& \mathbf{F}=\mathbf{T}_{\text {up }}+\mathbf{T}_{\mathrm{dw}}+\mathbf{G}+\mathbf{W}_{\mathrm{hub}}, \\
& \mathbf{M}=\mathbf{Q}_{\mathrm{up}}+\mathbf{Q}_{\mathrm{dw}}+\mathbf{r}_{\mathrm{Cup}} \times \mathbf{T}_{\mathrm{up}}+\mathbf{r}_{\mathrm{Cdw}} \times \mathbf{T}_{\mathrm{dw}} .
\end{aligned}
$$

where $\mathbf{r}_{\mathrm{Cdw}}$ and $\mathbf{r}_{\mathrm{Cup}}$ are the vectors from the CoG to the hub of the lower respectively upper rotor. In order to achieve a drift free flight, an alignment of the CoG with the rotor axis is necessary, therefore the two vectors are assumed to have only a $z$-component.

The rotor thrust vector $\mathbf{T}_{i}$ and torque vector $\mathbf{Q}_{i}$ we define as $\mathbf{T}_{i}=T_{i} \cdot \mathbf{n}_{T i}$ and $\mathbf{Q}_{i}=Q_{i} \cdot \mathbf{n}_{Q i}$, with $i=\{\mathrm{dw}, \mathrm{up}\}$ for the lower and upper rotor. In hover, the thrust magnitude $T_{i}$ of a rotor with radius $R$ can be defined as [8]

$$
T_{i}=c_{T i} \pi \rho R^{4} \Omega_{i}^{2}=c_{T i} k_{T} \Omega_{i}^{2},
$$

with the air density $\rho$, the rotational velocity $\Omega_{i}$ and the thrust coefficient $c_{T i}$. While the air density $\rho$ is assumed to be constant and the rotational velocity $\Omega_{i}$ depends on the motor input, the thrust coefficient $c_{T i}$ depends on different elements such as the rotor setup and the flow characteristics. However for a given rotor the thrust coefficient can be evaluated and due to its definition assumed to be constant over the used range of rotational rotor velocities. Another factor that influences the thrust coefficient is the inflow velocity of the rotor. This concerns mainly the lower rotor 
since it operates in the down wash of the upper rotor. Due to this down wash, the inflow velocity is increased and the thrust coefficient decreased [9]. This has to be considered by identifying the thrust coefficient of the lower rotor. The influence of the flow velocity due to movement in free flight are neglected in the model, since low inflow velocities do not significantly change the coefficients and high velocities are not foreseen for the dedicated missions.

The same assumptions are true for the torque coefficients $c_{Q i}$ that are used to describe the drag torque values $Q_{i}$ of the rotors and stabilizer bar:

$$
Q_{i}=c_{Q i} \pi \rho R^{5} \Omega_{i}^{2}=c_{Q i} k_{Q} \Omega_{i}^{2} .
$$

The direction of the thrust vector $\mathbf{n}_{i}$ is perpendicular to the TPP [6] and can be defined using tilt angles around the $\mathrm{x}$ - and $\mathrm{y}$ - axis as shown in Fig. 3.

Using two rotational transformations in series, the vector is expressed in the bodyfixed frame as

$$
\mathbf{n}_{T i}=\left[\begin{array}{c}
\cos \alpha \sin \beta \\
\sin \alpha \\
-\cos \alpha \cos \beta
\end{array}\right] .
$$

The direction of the rotor torque is equal to the $z$-axis, since the torque mainly acts on the rotor axis. It has to be considered that one rotor turns clockwise, while the other one turns counterclockwise.

$$
\mathbf{n}_{Q \text { up }}=\left[\begin{array}{l}
0 \\
0 \\
1
\end{array}\right], \mathbf{n}_{Q \mathrm{dw}}=\left[\begin{array}{c}
0 \\
0 \\
-1
\end{array}\right]
$$

The drag force value on the fuselage $W_{\text {hub }}$ due to the down wash of the rotors is small, but nevertheless considered in the model as a help for the identification (see

Fig. 3 Visualization of the tilted thrust vector with tilt angles $\alpha$ and $\beta$

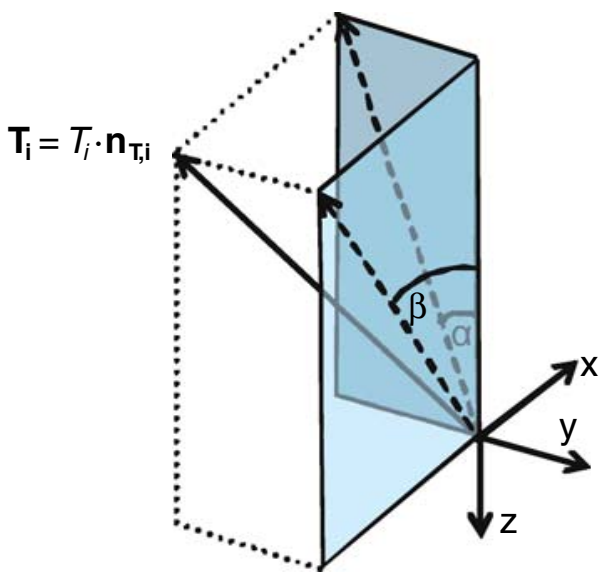


Section 4.2). It is assumed to be parallel to the $z$-axis and constant, since the down wash is nearly the same around hover:

$$
\mathbf{W}_{\text {hub }}=\left[\begin{array}{c}
0 \\
0 \\
W_{\text {hub }}
\end{array}\right]
$$

The last force is the gravitational force $\mathbf{G}$ caused by the mass of the helicopter. This force is always directed parallel to the $D$-axis (positive direction) of the inertial frame with the value $G=m g$. However the force is needed in the body-fixed frame, thus it has to be transformed using the transformation matrix from Eq. 2

$$
\mathbf{G}=\underline{\underline{\mathbf{A}}}_{B J} \cdot\left[\begin{array}{c}
0 \\
0 \\
m g
\end{array}\right]=m g \cdot\left[\begin{array}{c}
-\sin \theta \\
\cos \theta \sin \phi \\
\cos \theta \cos \phi
\end{array}\right]
$$

With all forces and moments defined, the next step is to model the dynamics of the stabilizer bar, swash plate and the electro motors.

\subsection{Stabilizer Bar and Swash Plate}

An important part of the system model is the stabilizer bar. In simple words this stabilization mechanism gives cyclic pitch inputs, similar to the swash plate, to the upper rotor to stabilize the helicopter in flight. The stabilizer bar has a high inertia and lags behind a roll or pitch movement of the fuselage as shown in Fig. 4. Through a rigid connection to the rotor, this time delay results in a cycling pitching of the rotor blades and therefore to a tilting of the TPP. If the stabilizer bar is adjusted correctly the thrust vector shows in the opposite direction of the roll or pitch movement causing a redress moment.

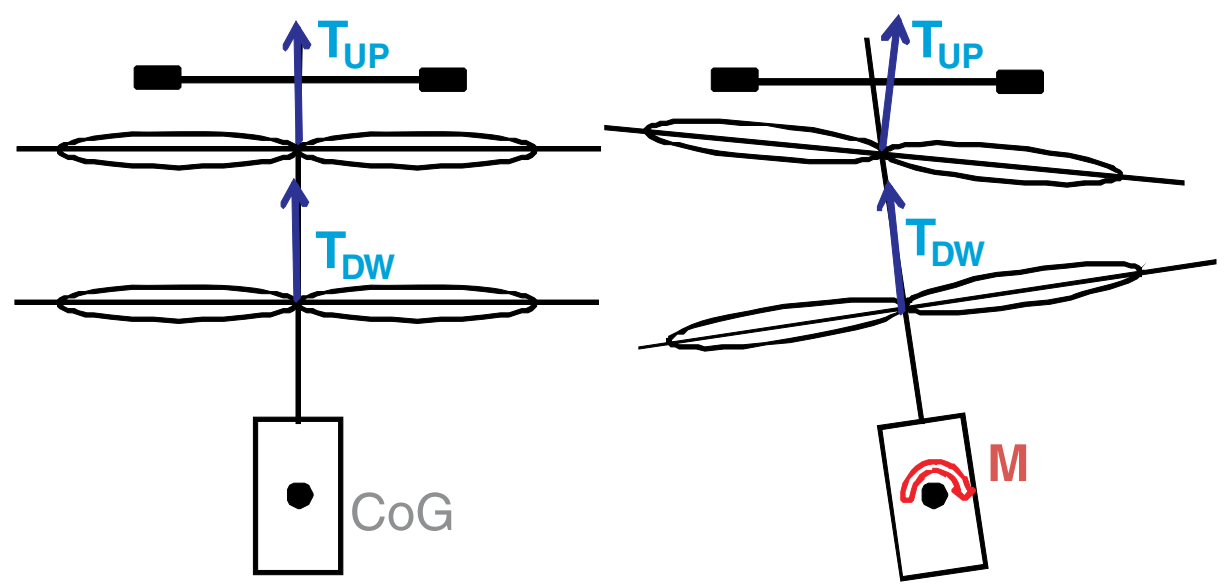

Fig. 4 The principle of the stabilizer bar. Due to the high inertia the stabilizer bar lags behind the roll/pitch movement, applies a cyclic pitch input to the rotor and creates a redress moment 
The stabilizer bar following the roll/pitch movement can be modeled as a first order element as

$$
\begin{aligned}
& \dot{\eta}_{\text {bar }}=\frac{1}{T_{\mathrm{f}, \text { up }}}\left(\phi-\eta_{\text {bar }}\right), \\
& \dot{\zeta}_{\text {bar }}=\frac{1}{T_{\text {f,up }}}\left(\theta-\zeta_{\text {bar }}\right),
\end{aligned}
$$

with angles $\eta_{\text {bar }}$ and $\zeta_{\text {bar }}$. The tilt angles of the thrust vector in the body-fixed frame is the difference between the two angles $\eta_{\text {bar }}$ and $\zeta_{\text {bar }}$ scaled by a factor $l_{\text {up }}$, since we are interested in the tilt angle of the TPP and not that of the stabilizer bar. Thus the equations for the tilting angles for the thrust vector are

$$
\begin{aligned}
& \alpha_{\text {up }}=l_{\text {up }}\left(\eta_{\text {bar }-\phi}\right) \\
& \beta_{\text {up }}=l_{\text {up }}\left(\zeta_{\text {bar }-\theta}\right) .
\end{aligned}
$$

The influence of the modeled stabilizer bar is shown in Fig. 5 where the reaction of the helicopter to an initial displacement in the roll angle is plotted. After a short time period the helicopter is back in the hover position.

The idea of the swash plate model is the same as for the stabilizer bar. The reaction from the servo input (PPM signal) to the change in the TPP is also modeled by a first order system. Hereby all the dynamics of the servos and rotor are covered by the time constant $T_{\mathrm{f}, \mathrm{dw}}$. The tilting angles of the lower rotor are modeled as

$$
\begin{aligned}
& \dot{\alpha}_{\mathrm{dw}}=\frac{1}{T_{\mathrm{f}, \mathrm{dw}}}\left(-l_{\mathrm{dw}} u_{\mathrm{serv} 2} \cdot \theta_{\mathrm{SPmax}}-\alpha_{\mathrm{dw}}\right), \\
& \dot{\beta}_{\mathrm{dw}}=\frac{1}{T_{\mathrm{f}, \mathrm{dw}}}\left(-l_{\mathrm{dw}} u_{\mathrm{serv} 1} \cdot \theta_{\mathrm{SPmax}}-\beta_{\mathrm{dw}}\right),
\end{aligned}
$$

with the time constant $T_{\mathrm{f}, \mathrm{dw}}$, scaling factor $l_{\mathrm{dw}}$, maximal swash plate tilting angle $\theta_{\text {SPmax }}$ and servo inputs $u_{\text {serv }, i}$.

Fig. 5 The reaction of the helicopter to an initial roll displacement of $20^{\circ}$ with the modeled stabilizer bar

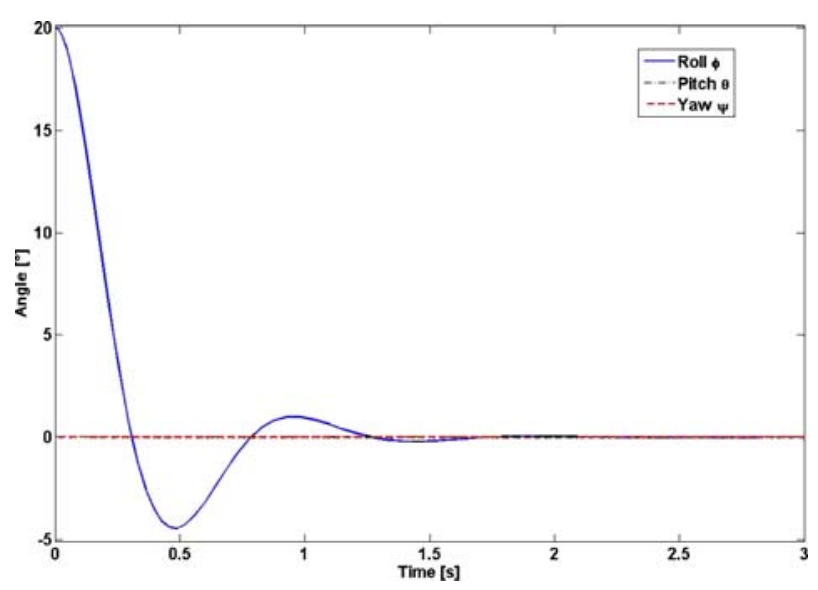




\subsection{Electro Motor}

The used BLDC motors are controlled by off-the-shelf motor controllers. They have the drawback that the motors rotational speed is not given and motor speed control is not possible. Instead of measuring the speed externally, for instance with an optical device, the motor dynamics are included directly in the model. This allows to use the model for every kind of motor without the help of any external sensor.

The differential equations for electro motors are well known [10] and can be simplified to an equation for the motor speed $\omega$ in the form of

$$
J_{\mathrm{mot}} \dot{\omega}=\frac{\kappa_{\mathrm{M}} U-\kappa_{\mathrm{M}} \kappa_{\mathrm{E}} \omega}{R_{\Omega}}-d_{R} \omega-M_{\mathrm{L}}
$$

with the moment of inertia $J_{\text {mot }}$, electrical and mechanical motor constants $\kappa_{\mathrm{E}}$ and $\kappa_{\mathrm{M}}$, the input voltage $U$, the electrical resistance $R_{\Omega}$, the friction coefficient $d_{R}$ and the external torque $M_{\mathrm{L}}$.

In the case of the helicopter, the external torque $M_{\mathrm{L}}$ is the drag torque value $Q_{i}$ of the rotor as shown in Eq. 9. Further, for the model the rotor speed is more important than the motor speed, thus the equation is expanded by the gears to obtain the final equation for the rotor speed $\Omega_{i}$

$$
J_{\text {drive }} \dot{\Omega}_{i}=\frac{\kappa_{\mathrm{M}} U_{\text {bat }} u_{\text {mot }, i}-\kappa_{\mathrm{M}} \kappa_{\mathrm{E}} i_{\text {gear }} \Omega_{i}}{i_{\text {gear }} R_{\Omega}}-d_{R} \Omega_{i}-\frac{c_{Q i} k_{Q} \Omega_{i}^{2}}{i_{\text {gear }}^{2} \cdot \eta_{\text {gear }}}
$$

with the gear ratio $i_{\text {gear }}$, the efficiency of the gear $\eta_{\text {gear }}$, the battery voltage $U_{\text {bat }}$ and the motor input $u_{\mathrm{mot}, i}$.

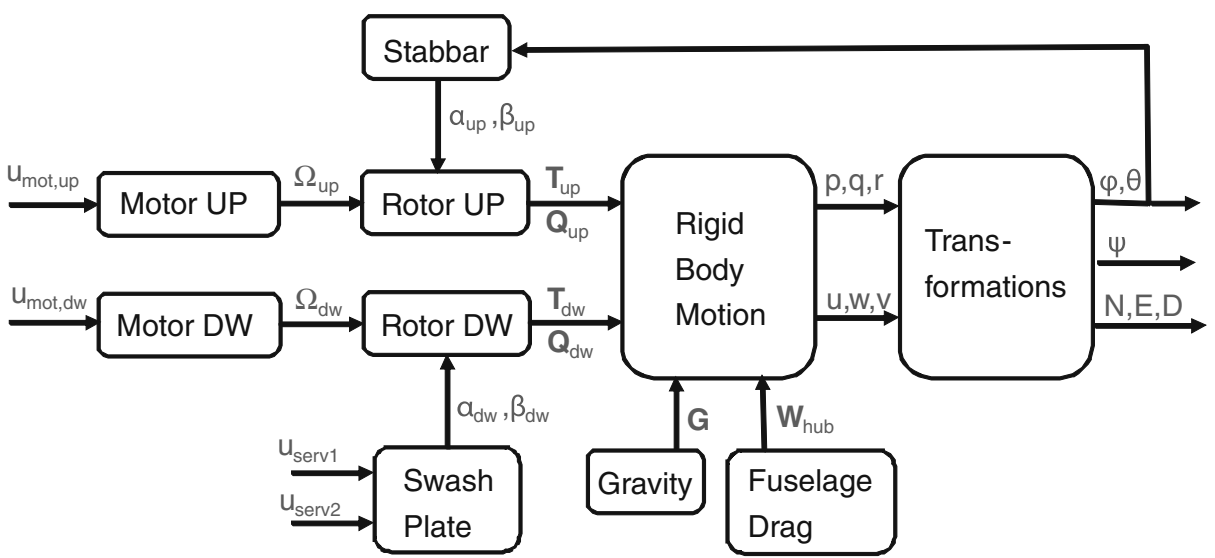

Fig. 6 muFly dynamic model block diagram 


\subsection{Model Summary}

With this set of differential equations, the nonlinear model is complete. The full model consists of 18 states and four inputs (two motors and two servos):

$$
\begin{aligned}
& x=\left[x, y, z, u, v, w, \phi, \theta, \psi, p, q, r, \alpha_{\mathrm{dw}}, \beta_{\mathrm{dw}}, \alpha_{\mathrm{up}}, \beta_{\mathrm{up}}, \Omega_{\mathrm{dw}}, \Omega_{\mathrm{up}}\right]^{T}, \\
& u=\left[u_{\mathrm{mot}, \mathrm{dw}}, u_{\mathrm{mot}, \mathrm{up}}, u_{\mathrm{serv} 1}, u_{\mathrm{serv} 2}\right]^{T}
\end{aligned}
$$

As an overview, the system is shown as a block diagram in Fig. 6. The equations of the complete system are given in the Appendix A.1.

\section{Parameter Identification}

After setting up the physical equations, the missing parameters have to be adjusted to the real helicopter. Without appropriate parameters, the best model is worthless.

\subsection{Mechanical Parameters}

The identification process is a non trivial task, and it is hard to find the correct parameters, especially since most of the parameters are coupled. In order to minimize the complexity of the identification on flight data, it is necessary to measure or estimate as many parameters as possible beforehand. While some parameters such as the mass, maximal swash plate angle, gear ratio, rotor diameter and body inertias can be easily measured or taken from the CAD design (all the equipment is modeled), it is more difficult to identify parameters such as the aerodynamical coefficients, electro motor constants or the time constants for the stabilizer bar and the swash plate.

\subsection{Aerodynamical Parameters}

The aerodynamical coefficients are calculated from the measurements on a coaxial rotor test-bench designed for blade optimization [11].

On this test-bench it is possible to identify the coefficients $c_{T i}$ and $c_{Q i}$ by measuring the torque and thrust of the lower and upper rotor. Even if it would have been possible, the torque coefficient of the stabilizer bar $c_{Q u p, s t a b}$ is not measured and only estimated instead. This parameter is identified later together with the fuselage drag $W_{\text {hub }}$ to achieve hovering condition with the recorded motor inputs for hovering. In other words, they are used as tuning parameters to correct the errors from the measurements to fulfill the equations in hover (index 'hov')

$$
\begin{array}{r}
T_{\text {up }, \text { hov }}+T_{\mathrm{dw}, \text { hov }}=G+W_{\text {hub }}, \\
Q_{\text {up,rot,hov }}+Q_{\text {up,stab,hov }}=Q_{\mathrm{dw}, \text { hov }},
\end{array}
$$

where as the drag torque of the upper rotor $Q_{\text {up }}$ is split in the rotor part $Q_{\text {up,rot }}$ and stabilizer bar $Q_{\text {up,stab. }}$ 


\subsection{Electro Motor}

The parameters for the low cost off-the-shell electro motors are not available, thus experimental data has to be used for the identification. Those motor measurements have been done by our partner CEDRAT [12] by applying a constant voltage, varying the external torques on the motors and measuring the rotational speed and current. The motor constants are identified using the stationary solution $\dot{\omega}=0$ of Eq. 17 and the Least-Square method (LS) [13]. Result plots for the identification are shown in Fig. 7.

\subsection{Prediction Error Method}

The remaining unknown parameters are identified dynamically using real flight data. Since the equations are not static anymore, a dynamic identification process has to be used. In this case, a linear Prediction Error Method (PEM) is chosen. This method is widely used in aeronautics and compares the measurement data vector $\mathbf{y}(t)$ with the predicted output of the dynamic model $\hat{\mathbf{y}}(t \mid t-1, \boldsymbol{\Theta})$ using the last $t-1$ measurements and the parameter vector $\Theta$ [14]. The difference between the measurement and the prediction

$$
\boldsymbol{\epsilon}(t, \boldsymbol{\Theta})=\mathbf{y}(t)-\hat{\boldsymbol{y}}(t \mid t-1, \boldsymbol{\Theta})
$$

is called the prediction error. The covariance matrix $\underline{\underline{\mathbf{R}}}_{N}(\boldsymbol{\Theta})$ is defined using all errors $\epsilon(1, \boldsymbol{\Theta}), \ldots, \epsilon(N, \boldsymbol{\Theta})$ :

$$
\underline{\underline{\mathbf{R}}}_{N}(\boldsymbol{\Theta})=\frac{1}{N} \sum_{t=1}^{N} \epsilon(t, \boldsymbol{\Theta}) \epsilon^{T}(t, \boldsymbol{\Theta})
$$

Fig. 7 Least square result plots for the electro motor parameter identification
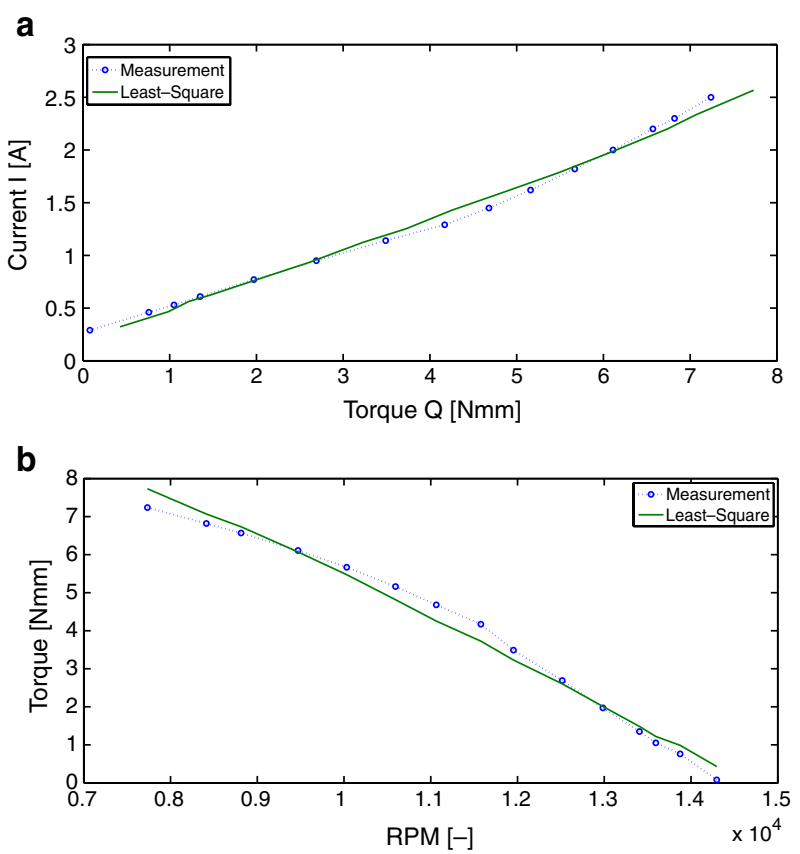
The wanted parameter vector $\boldsymbol{\Theta}_{\min }$ is now calculated such that the loss function $V_{N}(\Theta)$ is minimized:

$$
V_{N}(\boldsymbol{\Theta})=\operatorname{det}\left(\underline{\underline{\mathbf{R}}}_{N}(\boldsymbol{\Theta})\right) .
$$

For applying this method, the model has to be linearized around an operation point, in this case hover. Since only sensors for the attitude angles and the distance to the ground are mounted, the identification of $x / y$-dynamics of the model is not possible yet and is future work. Therefore the linearized model is reduced by the horizontal linear motion states, resulting in a state-space system of 14 states. It is almost impossible to identify all the parameters at once for such a high order system, therefore the system is split into four decoupled subsystems: pitch, roll, heave and yaw. As an example the subsystems for pitch and heave are shown in Eqs. 24 and 25, 26, respectively:

$$
\begin{aligned}
& A_{\mathrm{pitch}}=\left[\begin{array}{cccc}
0 & 1 & 0 & 0 \\
\frac{c_{T u p} k_{T} l_{\mathrm{up}} \Omega_{\mathrm{up}, 0}^{2} z_{\mathrm{up}}}{I_{y y}} & 0 & \frac{c_{T \mathrm{dw}} k_{T} \Omega_{\mathrm{dw}, 0}^{2} z_{\mathrm{dw}}}{I_{y y}}-\frac{c_{T u p} k_{T} l_{\mathrm{up}} \Omega_{\mathrm{up}, 0}^{2} z_{\mathrm{up}}}{I_{y y}} \\
0 & 0 & -\frac{1}{T_{\mathrm{f}, \mathrm{dw}}} & 0 \\
-\frac{1}{T_{\mathrm{f}, \mathrm{up}}} & 0 & 0 & \frac{1}{T_{\mathrm{f}, \mathrm{up}}}
\end{array}\right], \\
& B_{\text {pitch }}=\left[\begin{array}{c}
0 \\
0 \\
\frac{l_{\mathrm{dw}} \theta_{\mathrm{SPmax}}}{T_{\mathrm{f}, \mathrm{dw}}} \\
0
\end{array}\right] C_{\mathrm{pitch}}=\left[\begin{array}{llll}
1 & 0 & 0 & 0 \\
0 & 1 & 0 & 0
\end{array}\right] D_{\text {pitch }}=\left[\begin{array}{l}
0 \\
0
\end{array}\right] .
\end{aligned}
$$

The states and the input for the pitch subsystems are $x_{\text {pitch }}=\left[\theta, q, \beta_{\mathrm{dw}}, \beta_{\mathrm{up}}\right]^{T}$ and $u_{\text {pitch }}=u_{\text {serv } 1}$.

$$
\begin{aligned}
& A_{\text {heave }}=\left[\begin{array}{cccc}
0 & 1 & 0 & 0 \\
0 & 0 & -\frac{2 c_{T \mathrm{dw}} k_{T} \Omega_{\mathrm{dw}, 0}}{m} & -\frac{2 c_{T \mathrm{up}} k_{T} \Omega_{\mathrm{up}, 0}}{m} \\
0 & 0 & M_{\mathrm{dw}} & 0 \\
0 & 0 & 0 & M_{\mathrm{up}}
\end{array}\right] \text {, } \\
& B_{\text {heave }}=\left[\begin{array}{cc}
0 & 0 \\
0 & 0 \\
\frac{\kappa_{\mathrm{M}} U_{\mathrm{bat}}}{i_{\text {gear }} J_{\mathrm{drive}, \mathrm{dw}} R_{\Omega}} & 0 \\
0 & \frac{\kappa_{\mathrm{M}} U_{\mathrm{bat}}}{i_{\text {gear }} J_{\mathrm{drive}, \mathrm{up}} R_{\Omega}}
\end{array}\right] \\
& C_{\text {heave }}=\left[\begin{array}{llll}
1 & 0 & 0 & 0
\end{array}\right], D_{\text {heave }}=\left[\begin{array}{ll}
0 & 0
\end{array}\right],
\end{aligned}
$$




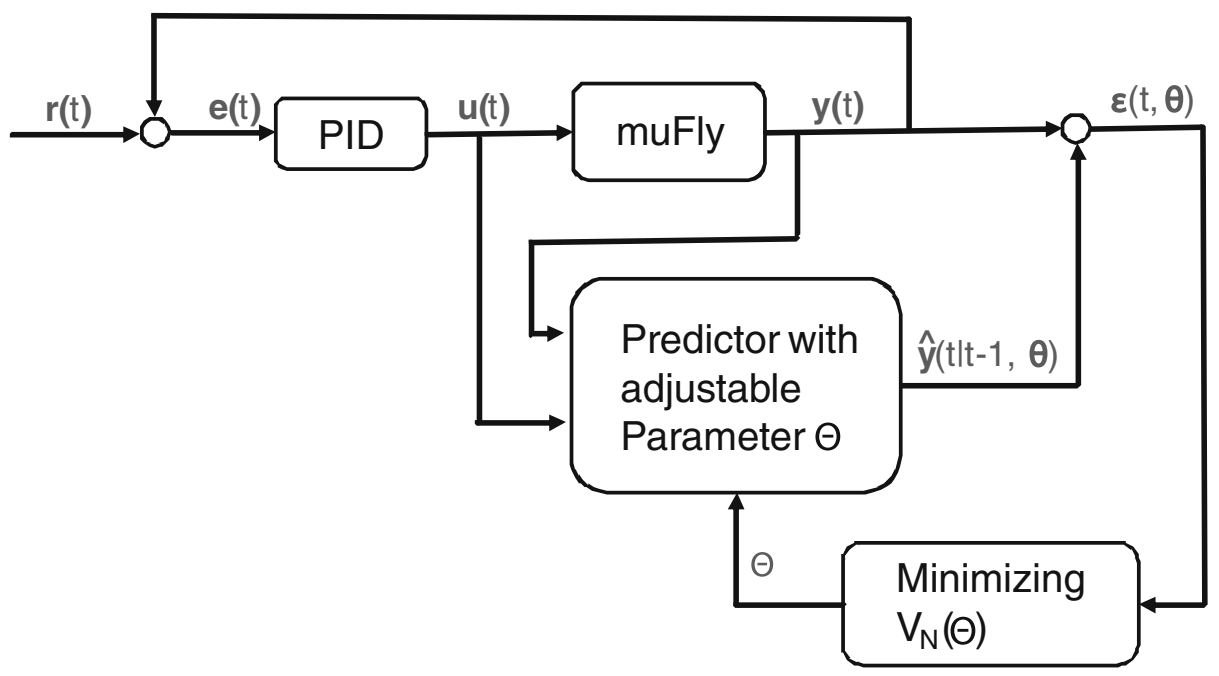

Fig. 8 Identification process block diagram
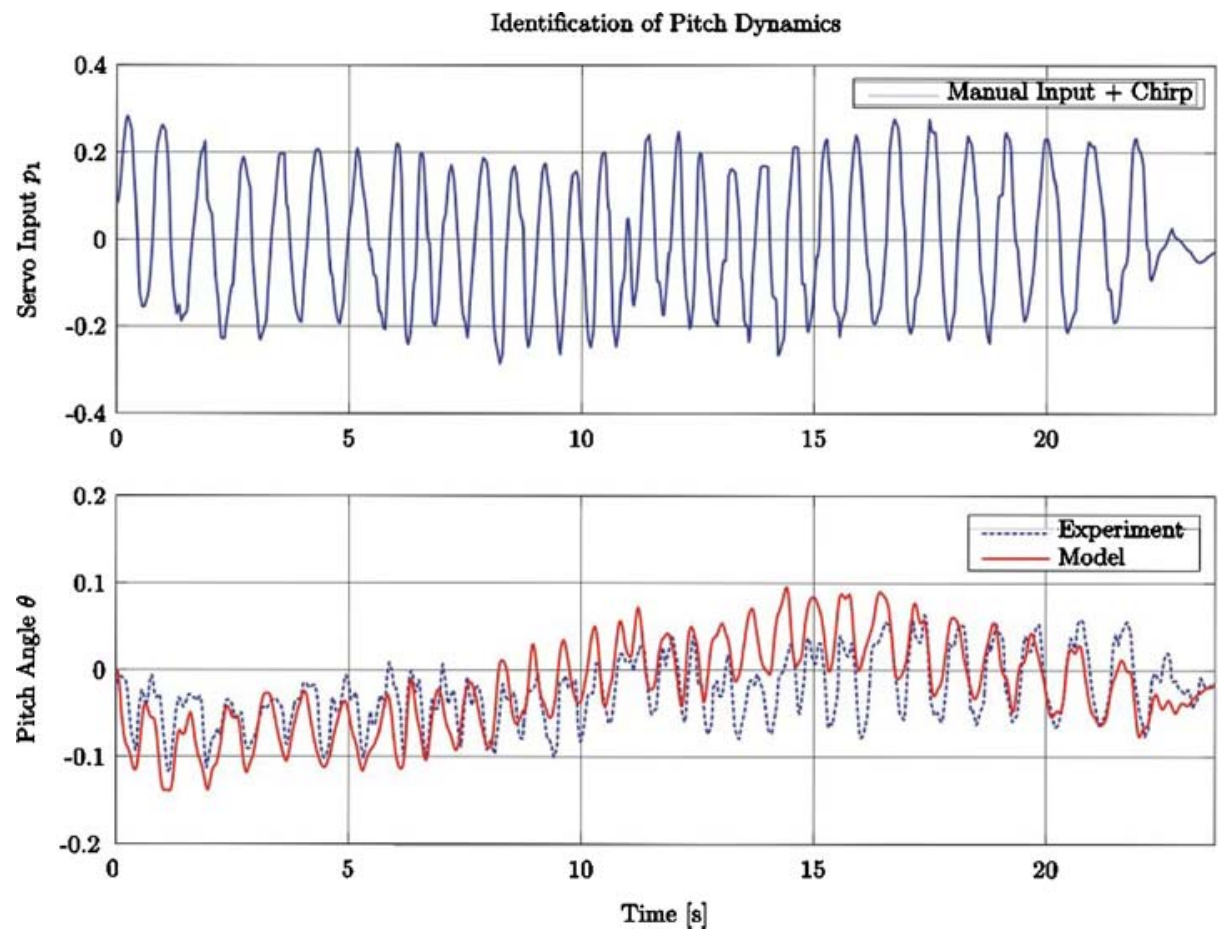

Fig. 9 Identification result of the pitch subsystem 
with

$$
\begin{aligned}
& M_{\mathrm{dw}}=\frac{1}{J_{\mathrm{drive}, \mathrm{dw}}} \cdot\left(-d_{R}-\frac{2 c_{Q \mathrm{dw}} k_{Q} \Omega_{\mathrm{dw}, 0}}{\eta_{\text {gear }} i_{\text {gear }}^{2}}-\frac{\kappa_{\mathrm{E}} \kappa_{\mathrm{M}}}{R_{\Omega}}\right), \\
& M_{\mathrm{up}}=\frac{1}{J_{\text {drive, up }}} \cdot\left(-d_{R}-\frac{2 c_{Q \mathrm{up}} k_{Q} \Omega_{\mathrm{up}, 0}}{\eta_{\text {gear }} i_{\text {gear }}^{2}}-\frac{\kappa_{\mathrm{E}} \kappa_{\mathrm{M}}}{R_{\Omega}}\right) .
\end{aligned}
$$

The states and the inputs for the heave subsystem are $x_{\text {heave }}=\left[z, w, \Omega_{\mathrm{dw}}, \Omega_{\mathrm{up}}\right]^{T}$ and the two motor inputs $u_{\text {heave }}=\left[u_{\text {mot,dw }}, u_{\text {mot,up }}\right]^{T}$.

The system for the roll dynamics is similar to the one of the pitch and the yaw dynamics similar to the heave. Therefore it is abandoned to show those systems here and are listed in the Appendix A.2.

The subsystems are identified after each other and it has to be considered that some parameters affect different subsystems. As an example the time constant $T_{\mathrm{f}, \mathrm{dw}}$ affects the roll and pitch subsystem. The time constant is identified on the roll system and kept constant in the pitch identification.

While the pitch and roll subsystems are easy to identify, the heave and yaw subsystems are very challenging due to the two poles at zero (critically stable). Due to this behavior it is difficult to identify the system over a long period of time without divergence, thus a good sequence has to be found. Further, the identification
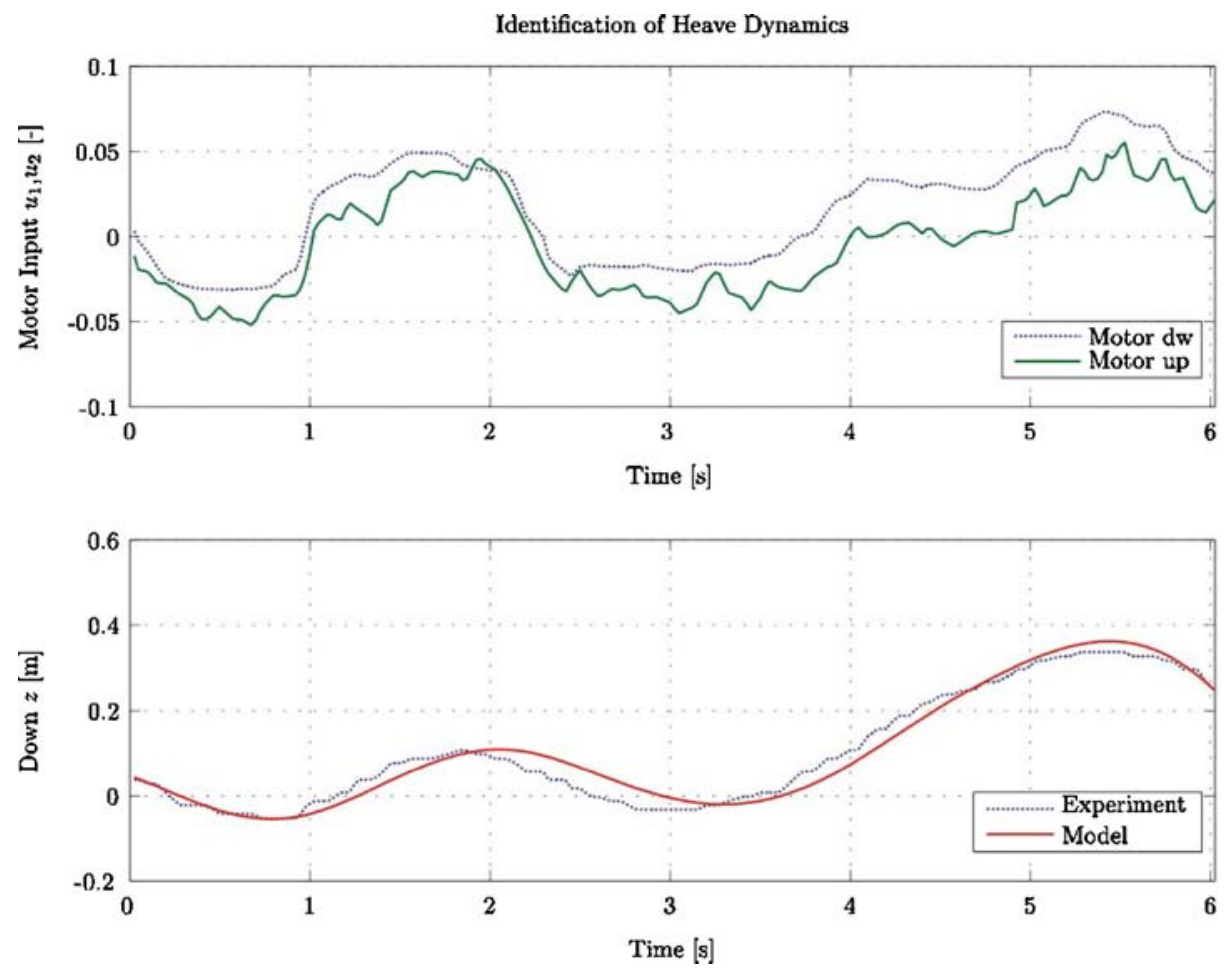

Fig. 10 Identification result of the heave subsystem 
process should start at the hover point and the inputs for hovering have to be determined from the measurements. Deviation in those inputs and in the unknown initial states leads to strong deviation in the result plots. Therefore the initial states and a deviation from the determined hover inputs are used as tuning parameters in the identification process.

\subsection{Data Generation}

For recording the flight data, the helicopter is steered by a pilot. In order to cover as much frequency bandwidth as possible, a chirp signal is generated and superimposed on the pilot input. However, the helicopter is not adequately controllable in open loop by a pilot. Therefore an additional PID controller is used to control attitude and help the pilot. Effectively, the pilot controls the set point values of the PID controller. The controller is not problematic for the parameter identification, since the actuator signals are recorded and send together with the sensor data. Hence the identification of the system is independent of the controller. An identification process overview is shown as a block diagram in Fig. 8. It shows the reference value input $\mathbf{r}(t)$ of the pilot as the input of the PID controller. The output of the controller $\mathbf{u}(t)$, the motor and servo inputs, is send to the helicopter and the respective subsystem, where a new output $\hat{\mathbf{y}}(t \mid t-1, \boldsymbol{\Theta})$ is predicted. This output is compared to the respective
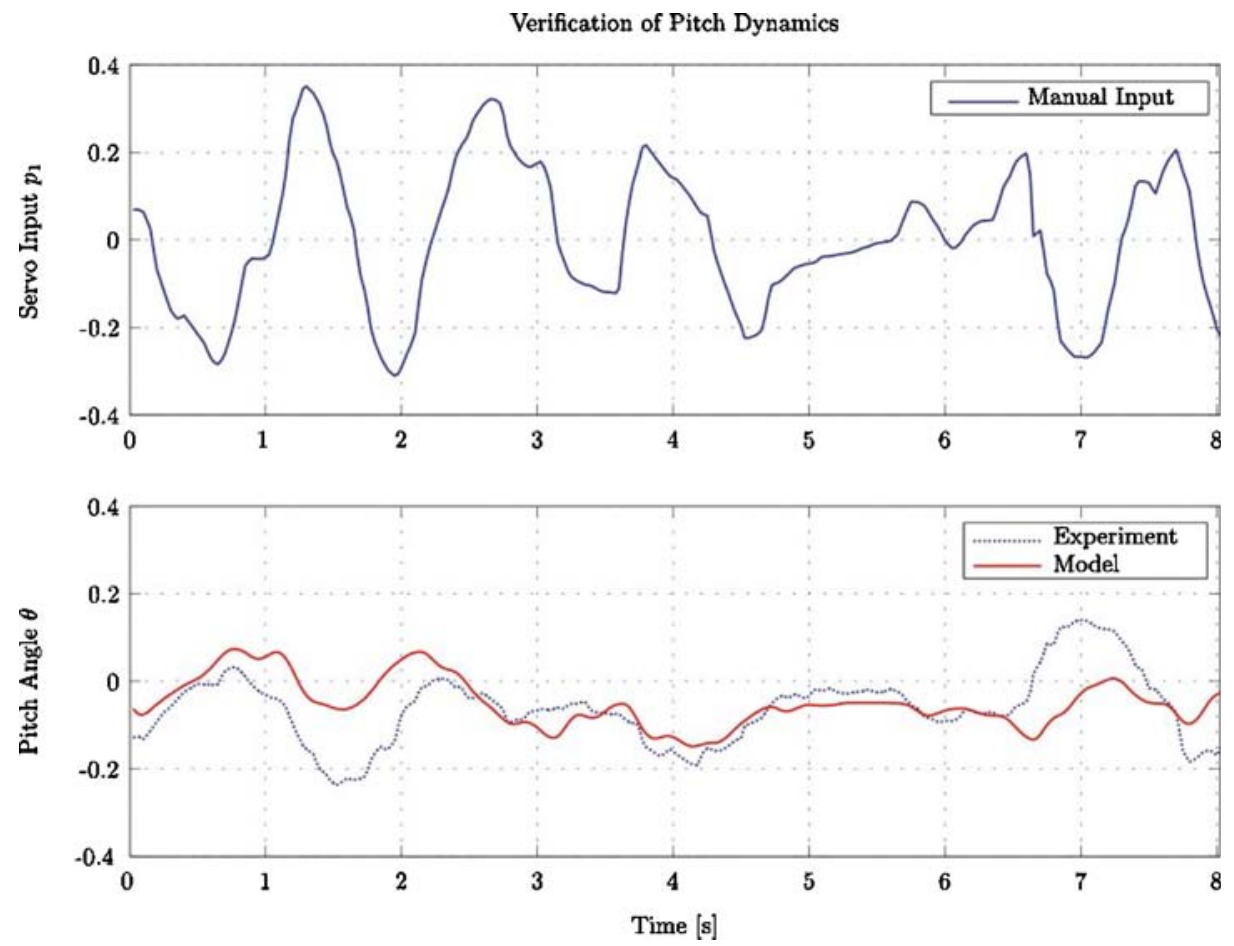

Fig. 11 Verification of the pitch subsystem 
sensor measurement $\mathbf{y}(t)$ and the error $\epsilon$ is build and minimized by adjusting the parameters $\boldsymbol{\Theta}$.

\section{Results}

The results for the identified subsystems for pitch and heave are shown in Figs. 9 and 10. In general the identified models show a good correlation with the real system. The main deviation is in the amplitude, which is not critical for the controller design, since those differences will be covered by the gain of the controller. More important is the phase, which shows that the subsystems are in the right dimensions. Well visible is the different time span of identification for the two subsystems. While the pitch subsystem can be identified over a time span of more than 20 seconds it is challenging to identify the heave system for a period longer than 10 seconds.

An identification alone is not sufficient, the model and the parameters have to be verified on an other flight sequence. For the verifications only the initial states and inputs are identified, all the parameters are taken from the previous identification. The results for the two subsystems are shown in Figs. 11 and 12. Those verification plots show again an acceptable correlation with the real platform, even if the performance is slightly worse. Here the model underestimates the amplitude,
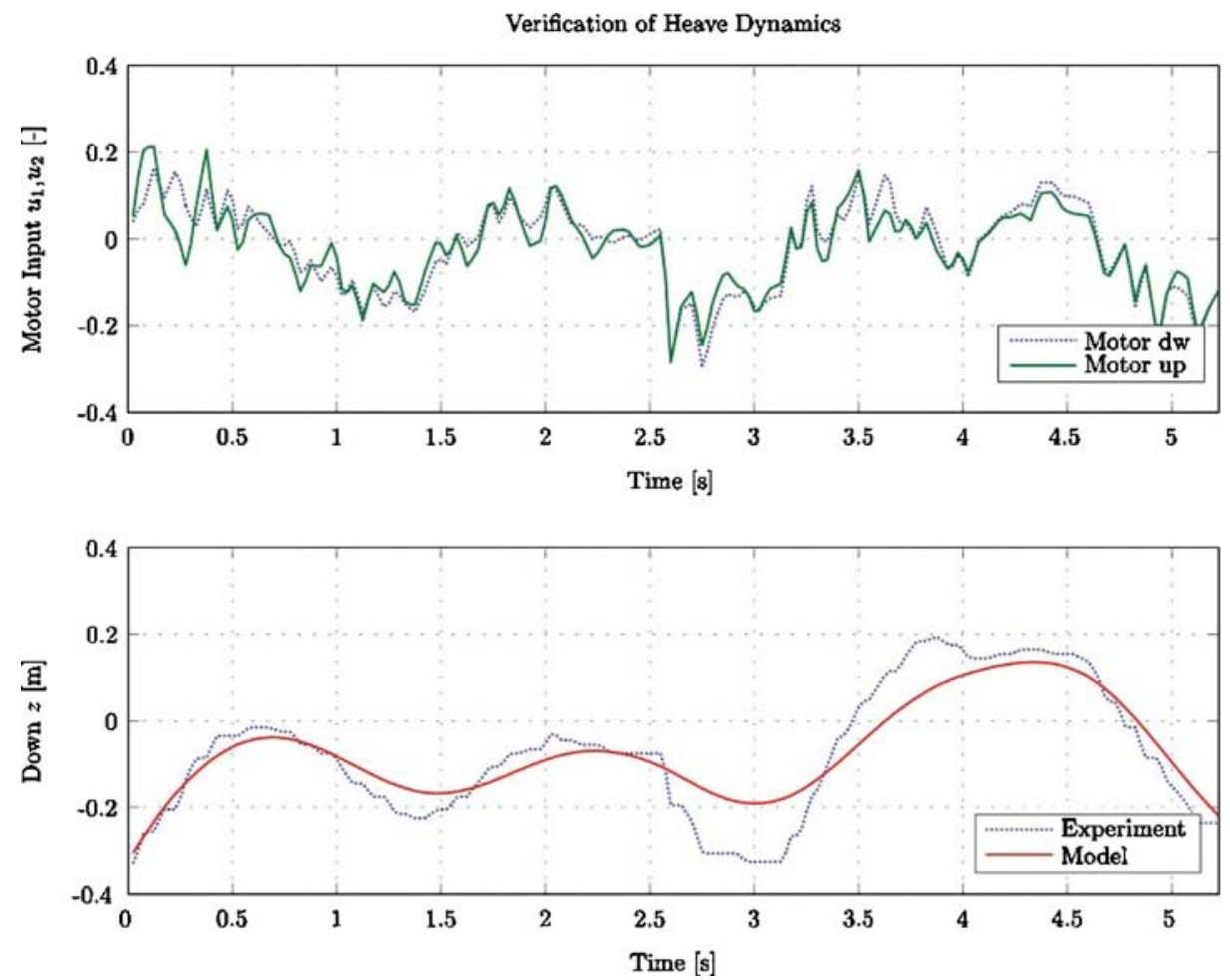

Fig. 12 Verification of the heave subsystem 
however, the more important phase matches again. All in all the identification and verification show good results and a proper controller design with those systems should be possible. A list of all the identified system parameters is given in Table 1 in the Appendix A.3.

\section{Summary and Future Work}

In this paper the development of a nonlinear model for the coaxial micro helicopter muFly is presented An appropriate model is essential for the controller design and simulations. The model is based on the rigid body dynamics where all the possible acting forces and moments are discussed. Further the dynamics of the mechanical devices such as the swash plate, electro motor and stabilizer bar are derived, resulting in a complete structured model.

However, a good model with bad parameters is worthless, hence those parameters have to be identified. While most parameters are measured from CAD data or on test benches, some parameters have to be identified on real flight data using identification methods. Since muFly will work mainly around the hover point, a linear prediction error method is chosen to be sufficient. The identification process is discussed and shown in detail, with specialties of using a PID controller to support the pilot or parameters which are not measured, but used as a correction of the measurements.

At last the results are presented showing the performance of the identified and verified models. The identified subsystems are now ready to be used for simulations and controller design. In future the models will be used to design and test altitude and attitude controllers using linear optimal controller techniques such as Linear Quadratic Gaussian (LGQ) or $\mathrm{H}_{\infty}$. Another idea is to identify the nonlinear model by using a nonlinear identification approach (e.g. based on neural networks), allowing to use nonlinear control techniques. After the completion of the position sensor the identified model will be used for a prediction of the position in a filter approach. The validation and verification of the $\mathrm{x} / \mathrm{y}$-dynamics of the model will be part of this process.

Acknowledgements The authors would like to thank Mr. P. Sax, Mr. F. Haenni, Mr. M. Buehler, Mr. D. Fenner and Mr. T. Baumgartner for their support. muFly is a STREP project under the Sixth Framework Programme of the European Commission, contract No. FP6-2005-IST-5-call 2.5.2 Micro/Nano Based Sub-Systems FP6-IST-034120. The authors gratefully acknowledge the contribution of our muFly project partners BeCAP at Berlin University of Technology, CEDRAT Technologies, CSEM, Department of Computer Science at University of Freiburg and XSENS Motion Technologies.

\section{Appendix}

A.1 Nonlinear Model

$$
\left[\begin{array}{l}
x \\
y \\
z
\end{array}\right]=\underline{\underline{\mathbf{A}}}_{B J} \cdot\left[\begin{array}{c}
N \\
E \\
D
\end{array}\right]
$$


$\underline{\underline{\mathbf{A}}}_{B J}=\left[\begin{array}{ccc}\mathrm{c} \theta \mathrm{c} \psi & \mathrm{c} \theta \mathrm{s} \psi & -\mathrm{s} \theta \\ -\mathrm{c} \phi \mathrm{s} \psi+\mathrm{s} \phi \mathrm{s} \theta \mathrm{c} \psi & \mathrm{c} \phi \mathrm{c} \psi+\mathrm{s} \phi \mathrm{s} \theta \mathrm{s} \psi & \mathrm{s} \phi \mathrm{c} \theta \\ \mathrm{s} \theta \mathrm{s} \psi+\mathrm{c} \phi \mathrm{s} \theta \mathrm{c} \psi & -\mathrm{s} \phi \mathrm{c} \psi+\mathrm{c} \phi \mathrm{s} \theta \mathrm{s} \psi & \mathrm{c} \phi \mathrm{c} \theta\end{array}\right]$

$\left[\begin{array}{c}\dot{\phi} \\ \dot{\theta} \\ \dot{\psi}\end{array}\right]=\underline{\underline{\mathbf{R}}}_{J B} \cdot\left[\begin{array}{c}p \\ q \\ r\end{array}\right]$

$\underline{\underline{\mathbf{R}}}_{J B}=\left[\begin{array}{ccc}1 & \frac{\mathrm{s} \phi \mathrm{s} \theta}{\mathrm{s} \theta} & \frac{\mathrm{s} \phi \mathrm{s} \theta}{\mathrm{s} \theta} \\ 0 & \mathrm{~s} \phi & -\mathrm{s} \phi \\ 0 & \frac{\mathrm{s} \phi}{\mathrm{s} \theta} & \frac{\mathrm{s} \phi}{\mathrm{s} \theta}\end{array}\right]$

$\left[\begin{array}{c}\dot{u} \\ \dot{v} \\ \dot{w}\end{array}\right]=\frac{1}{m} \mathbf{F}-\left[\begin{array}{c}p \\ q \\ r\end{array}\right] \times\left[\begin{array}{c}u \\ v \\ w\end{array}\right]$

$\left[\begin{array}{c}\dot{p} \\ \dot{q} \\ \dot{r}\end{array}\right]=\underline{\mathbf{I}}^{-1}\left(\mathbf{M}-\left[\begin{array}{c}p \\ q \\ r\end{array}\right] \times \underline{\underline{\mathbf{I}}}\left[\begin{array}{l}p \\ q \\ r\end{array}\right]\right)$

$\mathbf{F}=\left[\begin{array}{c}F_{x} \\ F_{y} \\ F_{z}\end{array}\right]=\mathbf{T}_{\text {up }}+\mathbf{T}_{\mathrm{dw}}+\mathbf{G}+\mathbf{W}_{\mathrm{hub}}$

$\mathbf{M}=\left[\begin{array}{c}M_{x} \\ M_{y} \\ M_{z}\end{array}\right]=\mathbf{Q}_{\mathrm{up}}+\mathbf{Q}_{\mathrm{dw}}+\mathbf{r}_{\mathrm{Cup}} \times \mathbf{T}_{\mathrm{up}}+\mathbf{r}_{\mathrm{Cdw}} \times \mathbf{T}_{\mathrm{dw}}$

$F_{x}=c_{T \mathrm{dw}} k_{T} \Omega_{\mathrm{dw}}^{2} \cos \beta_{\mathrm{dw}} \sin \alpha_{\mathrm{dw}}-c_{T \mathrm{up}} k_{T} \Omega_{\mathrm{up}}^{2} \sin \beta_{\mathrm{up}}-m g \sin \theta$

$F_{y}=c_{T \mathrm{dw}} k_{T} \Omega_{\mathrm{dw}}^{2} \sin \beta_{\mathrm{dw}}+c_{T \text { up }} k_{T} \Omega_{\mathrm{up}}^{2} \cos \beta \sin \alpha_{\mathrm{up}}+m g \cos \theta \sin \phi$

$F_{z}=-c_{T \mathrm{dw}} k_{T} \Omega_{\mathrm{dw}}^{2} \cos \alpha_{\mathrm{dw}} \cos \beta_{\mathrm{dw}}-c_{T \text { up }} k_{T} \Omega_{\text {up }}^{2} \cos \alpha_{\text {up }} \cos \beta_{\text {up }}$

$+W_{h u b}+m g \cos \theta \cos \phi$

$$
\begin{aligned}
& M_{x}=-z_{\mathrm{dw}} c_{T \mathrm{dw}} k_{T} \Omega_{\mathrm{dw}}^{2} \sin \beta_{\mathrm{dw}}-z_{\mathrm{up}} c_{T \mathrm{up}} k_{T} \Omega_{\mathrm{up}}^{2} \cos \beta_{\mathrm{up}} \sin \alpha_{\mathrm{up}} \\
& M_{y}=z_{\mathrm{dw}} c_{T \mathrm{dw}} k_{T} \Omega_{\mathrm{dw}}^{2} \cos \beta_{\mathrm{dw}} \sin \alpha_{\mathrm{dw}}-z_{\mathrm{up}} c_{T \mathrm{up}} k_{T} \Omega_{\mathrm{up}}^{2} \sin \beta_{\mathrm{up}} \\
& M_{z}=\left(c_{Q \mathrm{up}, \mathrm{rot}}+c_{Q \mathrm{up}, \mathrm{stab}}\right) k_{Q} \Omega_{\mathrm{up}}^{2}-c_{Q \mathrm{dw}} k_{Q} \Omega_{\mathrm{dw}}^{2}
\end{aligned}
$$

$$
\begin{gathered}
\alpha_{\text {up }}=l_{\text {up }}\left(\eta_{\text {bar }-\phi}\right) \\
\beta_{\text {up }}=l_{\text {up }}\left(\zeta_{\text {bar }-\theta}\right) . \\
\dot{\eta}_{\text {bar }}=\frac{1}{T_{\text {f,up }}}\left(\phi-\eta_{\text {bar }}\right), \\
\dot{\zeta}_{\text {bar }}=\frac{1}{T_{\text {f,up }}}\left(\theta-\zeta_{\text {bar }}\right),
\end{gathered}
$$




$$
\begin{gathered}
\dot{\alpha}_{\mathrm{dw}}=\frac{1}{T_{\mathrm{f}, \mathrm{dw}}}\left(-l_{\mathrm{dw}} u_{\mathrm{serv} 2} \cdot \theta_{\mathrm{SPmax}}-\alpha_{\mathrm{dw}}\right), \\
\dot{\beta}_{\mathrm{dw}}=\frac{1}{T_{\mathrm{f}, \mathrm{dw}}}\left(-l_{\mathrm{dw}} u_{\mathrm{serv} 1} \cdot \theta_{\mathrm{SPmax}}-\beta_{\mathrm{dw}}\right) \\
\dot{\Omega}_{\mathrm{dw}}=\frac{1}{J_{\mathrm{drive}, \mathrm{dw}}}\left(\frac{\kappa_{\mathrm{M}} U_{\mathrm{bat}} u_{\mathrm{mot}, \mathrm{dw}}-\kappa_{\mathrm{M}} \kappa_{\mathrm{E}} i_{\mathrm{gear}} \Omega_{\mathrm{dw}}}{i_{\mathrm{gear}} R_{\Omega}}-d_{\mathrm{R}} \Omega_{\mathrm{dw}}-\frac{c_{Q} k_{Q} \Omega_{\mathrm{dw}}^{2}}{i_{\mathrm{gear}}^{2} \cdot \eta_{\mathrm{gear}}}\right) \\
\dot{\Omega}_{\mathrm{up}}=\frac{1}{J_{\text {drive,up }}}\left(\frac{\kappa_{\mathrm{M}} U_{\mathrm{bat}} u_{\mathrm{mot}, \mathrm{up}}-\kappa_{\mathrm{M}} \kappa_{\mathrm{E}} i_{\mathrm{gear}} \Omega_{\mathrm{up}}}{\mathrm{i} R_{\Omega}}-d_{\mathrm{R}} \Omega_{\mathrm{up}}-\frac{c_{Q} k_{Q} \Omega_{\mathrm{up}}^{2}}{i_{\text {gear }}^{2} \cdot \eta_{\mathrm{gear}}}\right)
\end{gathered}
$$

\section{A.2 Linear Subsystems for Roll and Yaw}

Roll

$$
\begin{aligned}
& A_{\text {roll }}=\left[\begin{array}{cccc}
0 & 1 & 0 & 0 \\
\frac{c_{T \mathrm{up}} k_{T} l_{\mathrm{up}} \Omega_{\mathrm{up}, 0}^{2} z_{\mathrm{up}}}{I_{x x}} & 0-\frac{c_{T \mathrm{dw}} k_{T} \cdot \Omega_{\mathrm{dw}, 0}^{2} z_{\mathrm{dw}}}{I_{x x}}-\frac{c_{T u p} k_{T} l_{\mathrm{up}} \Omega_{\mathrm{up}, 0}^{2} z_{\mathrm{up}}}{I_{x x}} \\
0 & 0 & -\frac{1}{T_{\mathrm{f}, \mathrm{dw}}} & 0 \\
-\frac{1}{T_{\mathrm{f}, \mathrm{up}}} & 0 & 0 & \frac{1}{T_{\mathrm{f}, \mathrm{up}}}
\end{array}\right] \\
& B_{\text {roll }}=\left[\begin{array}{c}
0 \\
0 \\
\frac{l_{\mathrm{dw}} \theta_{\mathrm{SPmax}}}{T_{\mathrm{f}, \mathrm{dw}}} \\
0
\end{array}\right] C_{\text {roll }}=\left[\begin{array}{llll}
1 & 0 & 0 & 0 \\
0 & 1 & 0 & 0
\end{array}\right] D_{\text {roll }}=\left[\begin{array}{l}
0 \\
0
\end{array}\right]
\end{aligned}
$$

The states and the input are $x_{\text {roll }}=\left[\phi, p, \alpha_{\mathrm{dw}}, \alpha_{\mathrm{up}}\right]^{T}$ and $u_{\mathrm{roll}}=u_{\mathrm{serv} 2}$.

Yaw

$$
\begin{aligned}
& A_{\text {yaw }}=\left[\begin{array}{cccc}
0 & 1 & 0 & 0 \\
0 & 0 & -\frac{2 c_{Q \mathrm{dw}} k_{Q} \Omega_{\mathrm{dw}, 0}}{I_{z z}} & \frac{2 c_{Q \mathrm{up}} k_{Q} \Omega_{\mathrm{up}, 0}}{I_{z z}} \\
0 & 0 & M_{\mathrm{dw}} & 0 \\
0 & 0 & 0 & M_{\mathrm{up}}
\end{array}\right] \\
& B_{\text {yaw }}=\left[\begin{array}{cc}
0 & 0 \\
0 & 0 \\
\frac{\kappa_{\mathrm{M}} U_{\text {bat }}}{i_{\text {gear }} J_{\text {drive, dw }} R_{\Omega}} & 0 \\
0 & \frac{\kappa_{\mathrm{M}} U_{\text {bat }}}{i_{\text {gear }} J_{\text {drive, up }} R_{\Omega}}
\end{array}\right] \\
& C_{\text {yaw }}=\left[\begin{array}{llll}
1 & 0 & 0 & 0 \\
0 & 1 & 0 & 0
\end{array}\right] D_{\text {yaw }}=\left[\begin{array}{ll}
0 & 0 \\
0 & 0
\end{array}\right]
\end{aligned}
$$


with

$$
M_{\mathrm{dw}}=\frac{1}{J_{\text {drive }, \mathrm{dw}}}\left(-d_{\mathrm{R}}-\frac{2 c_{Q \mathrm{dw}} k_{Q} \Omega_{\mathrm{dw}, 0}}{\eta_{\text {gear }} i_{\text {gear }}^{2}}-\frac{\kappa_{\mathrm{E}} \kappa_{\mathrm{M}}}{R_{\Omega}}\right)
$$

and

$$
M_{\text {up }}=\frac{1}{J_{\text {drive,up }}}\left(-d_{\mathrm{R}}-\frac{2 c_{Q \text { up }} k_{Q} \Omega_{\text {up }, 0}}{\eta_{\text {gear }} i_{\text {gear }}^{2}}-\frac{\kappa_{\mathrm{E}} \kappa_{\mathrm{M}}}{R_{\Omega}}\right)
$$

The states and the inputs are $x_{\text {yaw }}=\left[\psi, r, \Omega_{\mathrm{dw}}, \Omega_{\mathrm{up}}\right]^{T}$ and $u_{\mathrm{yaw}}=\left[u_{\mathrm{mot}, \mathrm{dw}}\right.$, $\left.u_{\text {mot,up }}\right]^{T}$.

\section{A.3 The Identified Parameters}

\begin{tabular}{|c|c|c|c|c|}
\hline Parameter & Description & Identification & Value & Unit \\
\hline $\bar{m}$ & Mass & Measured & 0.095 & $\mathrm{~kg}$ \\
\hline$I_{x x}$ & Inertia around $x$-axis & CAD/PEM & $1.24 e^{-4}$ & $\mathrm{~kg} \mathrm{~m}^{2}$ \\
\hline$I_{y y}$ & Inertia around $y$-axis & $\mathrm{CAD} / \mathrm{PEM}$ & $1.30 e^{-4}$ & $\mathrm{~kg} \mathrm{~m}^{2}$ \\
\hline$I_{z z}$ & Inertia around $z$-axis & $\mathrm{CAD} / \mathrm{PEM}$ & $6.66 e^{-5}$ & $\mathrm{~kg} \mathrm{~m}^{2}$ \\
\hline$z_{\mathrm{dw}}$ & Distance CoG lower rotor hub & CAD & -0.051 & $\mathrm{~m}$ \\
\hline$z_{\text {up }}$ & Distance CoG upper rotor hub & Measured & -0.091 & $\mathrm{~m}$ \\
\hline$\Theta_{\mathrm{SP}, \max }$ & Maximal swash plate angle & Measured & 15 & $\circ$ \\
\hline$R$ & Rotor radius & Measured & 0.0875 & $\mathrm{~m}$ \\
\hline$c_{T \mathrm{dw}}$ & Thrust coefficient lower rotor & Test bench & 0.0115 & - \\
\hline$c_{T \text { up }}$ & Thrust coefficient upper rotor & Test bench & 0.0131 & - \\
\hline$c_{Q \mathrm{dw}}$ & Torque coefficient lower rotor & Test bench & 0.0018 & - \\
\hline$c_{Q \text { up, rot }}$ & Torque coefficient upper rotor & Test bench & 0.0019 & - \\
\hline$c_{Q \text { up,stab }}$ & Torque coefficient stabilizer bar & Hover/PEM & $3.58 e^{-5}$ & - \\
\hline$J_{\text {drive, dw }}$ & Drive train inertia (down) & $\mathrm{CAD} / \mathrm{PEM}$ & $1.914 e^{-5}$ & $\mathrm{~kg} \mathrm{~m}^{2}$ \\
\hline$J_{\text {drive,up }}$ & Drive train inertia (up) & $\mathrm{CAD} / \mathrm{PEM}$ & $9.78 e^{-6}$ & $\mathrm{~kg} \mathrm{~m}^{2}$ \\
\hline$\kappa_{\mathrm{E}}$ & Electrical motor constant & Least square & 0.0045 & $\mathrm{~V}^{-1} \mathrm{~s}^{-1}$ \\
\hline$\kappa_{\mathrm{M}}$ & Mechanical motor constant & Least square & 0.0035 & $\mathrm{Nm} \mathrm{A}^{-1}$ \\
\hline$d_{\mathrm{R}}$ & Motor friction & Least square & $5.2107 e^{-7}$ & $\mathrm{Nm} \mathrm{s}$ \\
\hline$R_{\Omega}$ & Resistance & Least square & 1.3811 & $\Omega$ \\
\hline$i_{\text {gear }}$ & Gear ratio & Measured & 1.5 & - \\
\hline$\eta_{\text {gear }}$ & Gear efficiency & Measured/PEM & 0.84 & - \\
\hline$W_{\text {hub }}$ & Drag force on the fuselage & Hover/PEM & 0.0108 & $\mathrm{~N}$ \\
\hline$T_{\mathrm{f}, \mathrm{dw}}$ & Following time upper rotor & PEM & 0.001 & $\mathrm{~s}$ \\
\hline$T_{\mathrm{f}, \mathrm{up}}$ & Following time upper rotor & PEM & 0.16 & $\mathrm{~s}$ \\
\hline$l_{\mathrm{dw}}$ & Linkage factor upper rotor & PEM & 0.41 & - \\
\hline$l_{\text {up }}$ & Linkage factor lower rotor & PEM & 0.83 & - \\
\hline
\end{tabular}

Table 1 Identified parameters

\section{References}

1. Prouty, R.W.: Helicopter Performance, Stability, and Control. PWS Engineering, Boston (1986)

2. Mettler, B.: Identification Modeling and Characteristics of Miniature Rotorcraft. Kluwer Academic, Dordrecht (2003)

3. Dzul, A., Hamel, T., Lozano, R.: Modeling and nonlinear control for a coaxial helicopter. In: IEEE International Conference on Systems, Man and Cybernetics, vol. 6 (2002) 
4. Chen, L., McKerrow, P.: Modelling the lama coaxial helicopter. In: Australasian Conference on Robotics \& Automation, Brisbane, Australia (2007)

5. Gerig, M.: Modeling, guidance, and control of aerobatics maneuvers of an autonomous helicopter. Ph.D. thesis, ETH Zurich (2008)

6. Watkinson, J.: The Art of the Helicopter. Elsevier, Amsterdam (2004)

7. Schafroth, D., Bermes, C., Bouabdallah, S., Siegwart, R.: Micro Helicopter Steering: Review and Design for the muFly Project. In: IEEE/ASME International Conference on Mechatronic and Embedded Systems and Applications (MESA), Beijing, China (2008)

8. Bramwell, A.: Helicopter Dynamics, 2nd edn. Butterworth Heinemann, Boston (2001)

9. Leishman, J.: Helicopter Aerodynamics, 2nd edn. Cambridge University Press, Cambridge (2006)

10. Mueller, G., Ponick, B.: Elektrische Maschinen Grundlagen elektrischer Maschinen. VCH, Weinheim (2006)

11. Schafroth, D., Bouabdallah, S., Bermes, C., Siegwart, R.: From the test benches to the first prototype of the muFly micro helicopter. J. Intell. Robot. Syst. 54(1-3), 245-260 (2008)

12. CEDRAT: CEDRAT technologies. http://www.cedrat.com (2009)

13. Schwarz, H.R., Kaeckler, N.: Numerische Mathematik. Stuttgart; Leipzig. Teubner, Wiesbaden (2004)

14. Moeckli, M.: Guidance and control for aerobatic maneuvres of an unmanned airplane. Ph.D. dissertation, ETH Zurich (2006) 